\title{
Vehicle routing in an urban area: environmental and technological determinants
}

\author{
K. Lewczuk ${ }^{1}$, J. Żak ${ }^{1}$, D. Pyza ${ }^{1}$ \& I. Jacyna-Gołda ${ }^{2}$ \\ ${ }^{1}$ Faculty of Transport, Warsaw University of Technology, Poland \\ ${ }^{2}$ Faculty of Process Engineering, \\ Warsaw University of Technology, Poland
}

\begin{abstract}
This paper discusses selected environmental and technological issues of routing freight vehicles in urban areas. The conditions of organizing distribution minimizing not only realization time but also the emission of harmful components of exhaust gases are described. Time-dependant traffic conditions characteristic for densely populated urban areas are defined and discussed according to ecological aspects. Theoretical information about $\mathrm{CO}_{2}$ emission in urban conditions are gathered and used to formulate the base of mathematical model of a Time-Dependant Vehicle Routing Problem with emission criteria function, as a version of Capacitated Vehicle Routing Problem.

The model comprises selected characteristics of urban transport infrastructure such as distances, speed limits, zones, or the effect of rush hours. It allows considering different types of vehicles according to the task requirements and emission characteristics, and changing traffic conditions.

The model is implemented onto a representation of a real transport network and solved for given data with two step-heuristics basing on a modified A-star algorithm and genetic programming. Appropriate results, future research directions and other potential uses are presented and discussed.

Keywords: emission, TDVRP, Time-Dependant Vehicle Routing Problem, CVRP, optimization, city logistics.
\end{abstract}

\section{Introduction}

Vehicle routing in urban areas, especially in city centres, is multifaceted logistics problem combining technological, economic, and environmental issues. 
Ecological and fast freight transport are factors improving the quality of life in cities, so the vehicles emission must be considered as a criteria of planning vehicle routes in urban transport network.

Urban conditions mean operating within the network of streets loaded by traffic dependent on rush hours and roads parameters. Municipal authorities restrict freight transport in the cities by limiting access, setting delivery hours or requiring high European Emission Standards for vehicles. Also the structure of urban transport network changes constantly. Small local reconfiguration like a closed street can cause significant disturbances in other areas. All these factors limit network transition time, influence emission of harmful components of exhaust gases - especially $\mathrm{CO}_{2}$, and induce a necessity of developing tools supporting decisions within vehicle routing in urban areas.

Lowering emission from freight transport can be stimulated on a central level by municipal authorities' decisions about traffic control and transportation policy. From the other hand it can be achieved by decreasing total mileage through rational vehicle routing, what is not only economically justified but also leads to perceiving transport companies as green. The first way requires global planning, while second one - presented in the paper - can be applied in operational planning of distributional companies.

Model presented in the paper concerns direct supplies from central depot to customers scattered in the city. Delivery time is related to traffic conditions and differs for particular hours and zones. While modelling urban conditions above elements will be considered:

1. The area is divided into zones according to traffic volume, flow capacity and accessibility. Zones can be outlined for city centre, historical old town, suburbs, residential area or business districts.

2. Characteristics of transport links: number of lines, speed limit, length, zone it belongs to, state (closed/open), type of allowed traffic (public busses, freight trucks, passenger cars).

3. Coefficient adjusting travel speed on each transport link according to its technical characteristics and according to the time-dependant conditions.

4. Type of vehicle (loading capacity, European Emission Standards).

The solution of the problem formulated in the paper is route minimizing time and vehicles emission level. Time-dependant conditions are considered, so the problem is a version of Time Dependent Vehicle Routing Problem (as proposed in [1]). Model takes into account indicators reducing speed and emission in relation to rated parameters of the transport network.

\section{On-road vehicles emission: $\mathrm{CO}_{2}$}

The most important vehicle ecological characteristic is on-road exhaust emission derived from the emission rate against travelled distance. Based on the airquality studies for cities like Beijing or Shanghai, the estimated contributions of vehicles emission to total air emissions were over $80 \%$ of $\mathrm{CO}, 50-80 \%$ of hydrocarbon and $40-75 \%$ of $\mathrm{NO}_{\mathrm{x}}$ [2]. Green House Gases and in particular $\mathrm{CO}_{2}$ 
are the most concerning as they have direct consequences on human health, and indirect ones (like depletion of the ozone layer) [3].

$\mathrm{CO}_{2}$ is emitted in direct proportion to fuel consumption, with a variation by type of fuel. For most vehicles, fuel consumption decrease as vehicle speed reaches 88 or $105 \mathrm{kph}$ and then begins to increase again. Also congestion has a great impact on vehicle emissions and fuel efficiency. In real driving conditions, there is a rapid nonlinear growth in emissions and fuel consumption as travel speeds fall below $38 \mathrm{kph}$ to $20 \mathrm{kph}$ or when it drops from $20 \mathrm{kph}$ to $8 \mathrm{kph}$ [4].

Every kilogram of carbon contained in fuel turns into $3.66 \mathrm{~kg}$ of $\mathrm{CO}_{2}$. Different fuels are characterized by different carbon content, so the emission rates are different. The complete combustion of $1 \mathrm{~kg}$ of diesel fuel produces about $3.11 \mathrm{~kg}$ of $\mathrm{CO}_{2}$, and combustion of methane produces $2.74 \mathrm{~kg}$ of $\mathrm{CO}_{2}$ [5]. Current research (i.e. [3-6]) show that emission pattern results from driving style, so it will dependant on speed and acceleration course as well as engine torque and rotation speed.

Alternative traffic control management strategy will likely change a vehicle's modal events in the traffic network and thus influence a vehicle's instantaneous speed profile. Different speed profiles will potentially result in different vehicle exhaust emission [6].

\section{CVRP with emission rates}

Time-Dependant Vehicle Routing Problem discussed in this paper is a version of a Capacitated VRP based on relaxed Travelling Salesman Problem, for which constrain restricting visiting nodes only once is omitted. In city conditions, passing the same crossroad more than once is common. Founding shortest paths in transportation networks leads to shortening transport cycles and lowering costs, so it also can be adapted to lower freight vehicles emission.

The VRP with emission as a criterion is discussed in the literature. Bektaş and Laporte [3] present the Pollution-Routing Problem expanding classical VRP, and discuss economies of environmental-friendly vehicle routing. They state that contrary to the VRP, the PRP is significantly more difficult to solve to optimality. Kuo and Wang [7] present approach to optimize the routing plan with minimizing fuel consumption with a Tabu Search and prove that this approach leads to better economic results than minimizing distances only. A similar problem is considered by Suzuki [8] who develops an approach to the timeconstrained, multiple-stop, VRP lowering fuel consumption and pollutants emission by minimizing distance travelled with full load on board. Above two papers indirectly deal with emission minimizing. Kara et al. [9] propose a new cost function based on distance and load of the vehicle for the CVRP. The vehicle-routing problem with this load-based cost objective is called the Energy Minimizing VRP. Apaydin and Gonullu [10] deal with route optimization in solid waste collection in order to decrease harmful diesel emission. They propose using Geographical Information System (GIS) elements such as numerical pathways to problem identification - as it was assumed in this paper. 
Another issue is constructing VRP models, especially for time-dependent traffic conditions in cities. The area is well recognized (i.e. state-of-the-art on models [11]) and known methods can be used. Exemplary VRP model with single depot (Cargo Consolidation Centre) in urban conditions was presented by Pyza [12]. Model reduces cargo traffic in urban area by coordinating traffic flows and eliminating empty runs. Similar approach is presented by Jacyna [13]. Jachimowski et al. [14] and Pyza [15] consider routes planning problem with heterogeneous suppliers demand. Cornillier et al. [16] discuss the Petrol Station Replenishment Problem with Time Windows focusing on determining routes, and schedules. Chen et al. [17] formulate real-time time-dependent VRP with Time Windows with regard to time-dependent travel times. Similar approach is adapted to the problem discussed in this paper. The exact Time Dependant VRP is formulated by Malandraki and Daskin [1] or Figliozzi [4].

VRP models can be solved according to different criteria functions; Jachimowski and Żak [18] formulate VRP with Heterogeneous Customers Demand according to external transportation costs. Jacyna and Kłodawski [19] discuss the problem in aspect of transport co-modality, similarly as Żak et al. [20], Lewczuk et al. [21]. Lewczuk and Wasiak [22] evaluate TSP solving in aspect of cost sharing. Emission as criteria is used in different ways in [3, 7-9, 10].

Adduced papers include methods, elements of methods and approaches to TDVRP and CVRP with emission, but do not comprise the approach as it is presented in this paper.

\section{Formal model}

\subsection{Transportation network}

Proposed model describes the issue of setting best possible route in timedependant traffic conditions to minimize vehicle's emission and serve the clients on the demanded quality level. Transportation process is realized in transport network of the city for which traditional flow-arc formulation is adopted:

$$
S=\left\langle G, F_{L}\right\rangle
$$

where:

$S \quad$ - transport network,

$\boldsymbol{G}$ - graph of network structure; $\boldsymbol{G}=\langle\boldsymbol{W}, \boldsymbol{L}\rangle$,

W - set of nodes,

$\boldsymbol{L}$ - set of permitted transport links (edges) between nodes.

$\boldsymbol{F}_{\boldsymbol{L}} \quad$ - set of functions defining edge transition parameters.

Graph G contains details of transportation network structure. Each link between two nodes representing adjacent crossroads in the real network, in most cases, is composed of few segments representing particular sections of transport link (Figure 1). This assumption allows using Geographic Information System to precise modelling and applying proposed approach in practice.

The links between nodes correspond to sections that street can be divided into. Each section is represented by pair of edges of opposite directions with 
known length, number of lines, speed limit, allowed types of vehicles to move on, and variable hourly capacity. The hourly capacity changes in a reaction to traffic conditions and is correlated to the zone it belongs to. It is significant especially in rush hours when smooth movement is impeded. Functions $\mathbf{F}_{\mathbf{L}}$ on the edges can be changed to simulate real situations like closed street or restricted access. All nodes in graph allow turning back.

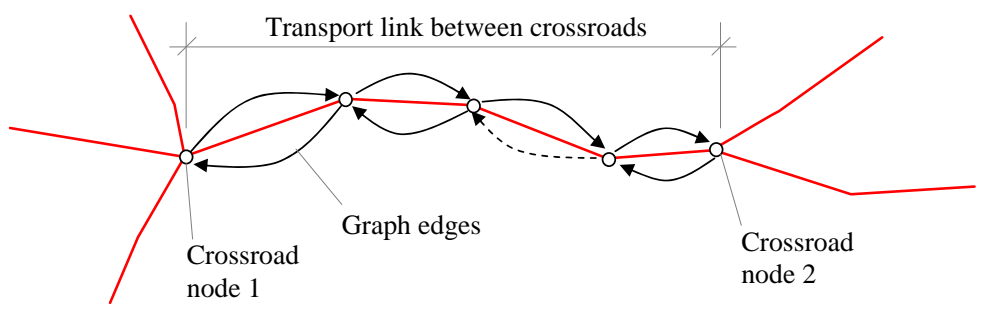

Figure 1: The representation of transport link sections in graph.

The set of clients' orders is known. Clients are attributed to the nodes. Different types of vehicles can be used. Vehicles start and finish in central depot. The daily work time can't exceed the assumed value.

\subsection{Indexes and parameters}

Let the following indexes be defined:

$$
\begin{array}{lll}
i, j, k & \text { - numbers of graph nodes; } & i, j, k \in \boldsymbol{W} \\
i & \text { - client number; } & i \in \boldsymbol{C}, \boldsymbol{C} \\
s & \text { - vehicle type; } & s \in \boldsymbol{S} \\
t & \text { - time moment; } & t \in \boldsymbol{T} \\
e & \text { - emission component; } & e \in \boldsymbol{E} \\
m & \text { - zone number; } & m \in \boldsymbol{M} \\
\boldsymbol{L}^{m} & \text { - set of edges assigned to } m \text {-th zone; } & \boldsymbol{L}^{m} \subset \boldsymbol{L} \\
h & \text { - number of time intervals; } & h \in \boldsymbol{H} \\
\varepsilon & \text { - vehicle emission standards (EURO); } & \varepsilon \in \boldsymbol{E} \boldsymbol{U}
\end{array}
$$

and the model parameters:

$\mathrm{i}=0$ - central depot;

$\mathrm{h}(\mathrm{t}) \quad$-assignation of $\mathrm{t}$-th time moment to $\mathrm{h}$-th time interval;

$\mathrm{T}_{\mathrm{c}} \quad$ - maximal daily work time set for driver;

$\alpha(i)$ - binary value, equal to 1 when i-th node has attributed client.

$v_{i, j} \quad$ - average speed of travel between nodes $i$ and $j$;

$v_{i, j}^{\max } \quad$-speed limit between nodes $\mathrm{i}$ and $\mathrm{j}$.

$\mathrm{g}_{\mathrm{s}, \mathrm{e}} \quad$ - average level of e-th type emission by s-th type vehicle;

$w_{s, e, j}^{h(t)}$-coefficient adjusting amount of $e$-th type emission for $s$-th type vehicle in $h$-th interval on edge from $i$ to $j$; 
$d_{i, j} \quad$ - distance between nodes $i$ and $j$, where $d_{i, j}=d_{i, j} \vee d_{i, j} \neq d_{i, j}$;

$k_{i, j}^{h(t)} \quad$ - coefficient adjusting speed between nodes $i$ and $j$ in $h$-th interval;

$\Gamma_{i}^{-1} \quad$ - set of predecessors of $\mathrm{i}$-th node;

$\Gamma_{i} \quad$ - set of consequents of $i$-th node;

$\mathrm{q}_{\mathrm{i}} \quad$ - ordered amount of material for client attributed to $\mathrm{i}$-th node, if client is not attributed to $i$-th node $\mathrm{q}_{\mathrm{i}}=0$;

$\tau_{\mathrm{i}} \quad$ - time of loading operations in $\mathrm{i}$-th node if client is attributed to the node, if client is not attributed to $i$-th node $\tau_{\mathrm{i}}=0$,

$\mathrm{t}_{0} \quad$ - time of loading operation in $\mathrm{i}$-th node if client is attributed to the node;

$\beta_{\varepsilon, \mathrm{m}}-$ minimal standard emission required to enter m-th zone.

$\mathrm{Q}_{\mathrm{s}} \quad$ - loading capacity of s-th type vehicle.

$\mathrm{n}_{\varepsilon, \mathrm{s}} \quad$ - emission standard of s-th type vehicle.

The decision variable is:

$x_{i, j}^{s, t} \quad-a$ binary variable equal to 1 if and only if s-th type of vehicle is to be present on edge between nodes $i$ and $j$ in $t$-th moment.

\subsection{Criteria function and constrains}

Minimize:

$$
\forall e \in \boldsymbol{E} \forall s \in \boldsymbol{S} \sum_{(i, j) \in \boldsymbol{L}} \sum_{t \in \boldsymbol{T}} x_{i j}^{s, t} q_{s, e} w_{s, e, j j}^{h(t)} \frac{d_{i j}}{V_{i j} k_{i j}^{h(t)}}
$$

while constrains:

$$
\begin{gathered}
\forall i \in \boldsymbol{W} \forall s \in \boldsymbol{S} \sum_{t \in \boldsymbol{T}} \sum_{j \in \Gamma_{t}^{-t}} x_{j i}^{s, t}-\sum_{t \in \boldsymbol{T}} \sum_{k \in \Gamma_{t}} x_{i k}^{s, t}=0 \\
\forall(i, j) \in \boldsymbol{L} \forall s \in \boldsymbol{S} \forall h \in \boldsymbol{H} \forall t \in \boldsymbol{T} x_{i j}^{s, t} v_{i j} k_{i j}^{h(t)} \leq v_{i j}^{\max } \\
\forall s \in \boldsymbol{S} \sum_{i \in \boldsymbol{W}} \sum_{t \in \boldsymbol{T}} x_{0 i}^{s, t} q_{i}+\sum_{i \in \boldsymbol{W}} \sum_{j \in \boldsymbol{W}: i \neq j} \sum_{t \in \boldsymbol{T}} x_{i j}^{s, t} q_{i} \leq Q_{s} \\
\forall(i, j) \in \boldsymbol{L}^{m} \forall m \in \boldsymbol{M} \forall t \in \boldsymbol{T} x_{i j}^{s, t} \leq \operatorname{sgn}\left(n_{\varepsilon, s}-\beta_{e, m}\right)+1 \\
\forall s \in \boldsymbol{S} \sum_{t \in \boldsymbol{T}} \sum_{i \in W} x_{0 i}^{s, t}-\sum_{t \in \boldsymbol{T}} \sum_{j \in W} x_{j 0}^{s, t}=0 \\
\forall s \in \boldsymbol{S} \sum_{(i, j) \in \boldsymbol{L}} \sum_{t \in \boldsymbol{T}} x_{i j}^{s, t}\left(\frac{d_{i j}}{v_{i j} k_{i j}^{h(t)}}+\tau_{i}\right) \leq T_{c} \\
\forall i \in \boldsymbol{C} \forall s \in \boldsymbol{S} \sum_{(i, j) \in \boldsymbol{L}} \sum_{t \in \boldsymbol{T}} x_{i j}^{s, t} \geq 1 .
\end{gathered}
$$

must be kept.

The model is then (1) subject to (2-7), and (8). In this formulation, the objective function minimizes the total emission of e-th type of harmful factor by s-th type of vehicle while realizing customer order in time-dependant traffic conditions. Constraint (2) stipulates that each vehicle visiting i-th node (as 
transitional) will leave this node. Constraint (3) ensures that speed limits will not be exceeded. Constraint (4) keeps s-th type vehicle loading capacity not exceeded. Constrain (5) states that vehicles with low emission standard will not enter zones requiring high standards. Constrain (6) ensures that vehicle will came back to the starting point at the end of the route. Constraint (7) ensures that regular and overtime hours lie within the allowable limits. Constrain (8) stipulates that every client will be serviced.

\subsection{Adjusting factors}

The difficulty is in finding factors $w_{s, e, i j}^{h(t)}$ and $k_{i j}^{h(t)}$. The second one can be estimated by empirical measures of traffic in reliable cross-sections of transportation network or can be calculated. Examples of calculation methods can be found in $[23,24]$.

Adjustment of coefficient $w_{s, e, i j}^{h(t)}$ must reflect vehicle's speed profile, especially instantaneous speed, acceleration, type of fuel, engine power and power torque. Necessary methodology is present in [2, 3, 5, 6, 25].

At this stage of research it is assumed that emission of $\mathrm{CO}_{2}$ is proportional to fuel consumption and dependent on engine Euro Emission Standard. The methodology presented in [7] is applied. Fuel consumption is then a function of instantaneous speed profile and type of vehicle.

\section{Solving}

Practical apply of proposed mathematical model demands algorithm finding sufficient solution in acceptable time. According to that a two-step heuristics was developed. Proposed approach is based on genetic programming for frame searching, and A-star algorithm for pathfinding. A-star algorithm is modified to meet time-dependant traffic conditions defined in the model. The general idea of heuristics is to solve classic TSP deprived of constrain limiting visiting nodes only once. Efficient traversable paths between nodes are plotted by modified Astar algorithm with regard to emission level and time.

\subsection{A-star modification}

A standard version of A-star algorithm proposed by Hart et al. in [26] searches for least-cost path by evaluating only one cost measure at the time, like travel time or distance. Vehicle's emission level is subjected to traffic conditions and differs when average speed and acceleration are changing. The vehicle's emission and movement characteristics in most cases are described by non-linear functions, so A-star was adapted to deal with that problem.

The distance-plus-cost heuristic function in A-star is a sum of two elements: the path-cost function from starting to current node, and admissible heuristic estimation of cost of traveling from current to finish node. The path-cost function, unlike the classic formulation, calculates vehicle emission according to 
traffic conditions in current moment quantified to time intervals $h(t)$. It also calculates the time of previous travel to find out in which time interval the next stage will start, so traffic constrains for next stage can be found.

The heuristic estimation of the cost of remaining travel also must consider time-dependant conditions. Heuristics firstly plots straight line connecting the coordinates of current node with coordinates of finish node. Then registered intersections with graph edges are cutting plotted line into segments that are to be temporary edges used only to estimate cost of remaining travel. Transitions costs for these new edges are adopted from genuine edges with rule that new edge has a cost of edge with which it crosses at the end (Figure 2).

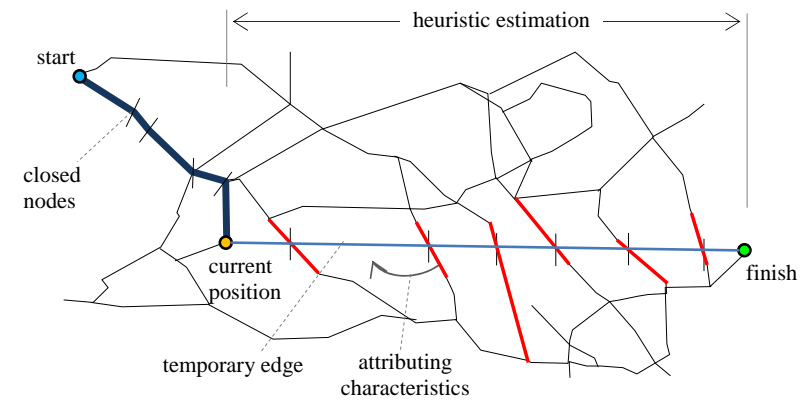

Figure 2: A-star modification for time-dependant traffic conditions.

Of course transition costs change in time, so attributing cost from genuine edges must take into account the time of travel through new edges. This time is estimated with using the information about zone it belongs to, actual time and coefficient found while calibrating. Finally, using modified A-star algorithm results in calculating for each considered pair of nodes in graph the total emission and traveling time. Emission will be counted by criteria function and time will be an input parameter for next pair of nodes.

\subsection{Genetic programming}

TDVRP based on relaxed TSP can be solved efficiently for static conditions determining transition cost. Taking into account variable conditions and the fact that total cost is not expressed in natural measure like time or distance the genetic programming was applied to solve the problem on the second stage.

In used genetic algorithm chromosome encodes the sequence of clients to be visited as a series of their numbers. Two types of crossover operators are used: 1/ one cut-point, and 2/ PMX with two cut-points. Four different policies of selecting pairs of parents were considered: $1 /$ sequentially best with best, $2 /$ sequentially with random, 3/ sequentially best with worst and 4/ random with random. The mutation coefficient changes during calculation. At the beginning it keeps reference value, but together with the progress of calculation it increases. Thanks to that in the first stage recombination has a dominant role, while in the 
second stage of calculation extensive mutation of best individuals usually leads to improvement. Experiments were carried for fixed number of generations (3,000 to 10,000) embracing constant number of individuals (30 to 70). The new generation was composed of 10 to $20 \%$ of best parents and 50 to $60 \%$ of best fitted offspring. The rest was randomly selected.

\section{The example}

\subsection{Network}

Preliminary experiments were carried for transportation network of a city with the population size of 180,000 people. Major roads are represented by graph composed of more than 4,700 edges and about 1,800 nodes. Each edge is described by length, number of lines, speed limit, type of allowed traffic (trucks, busses, or passenger cars), zone that it belongs to, and flow capacity. Flow capacity is estimated for 27 time intervals, 20 minutes each starting from $5 \mathrm{am}$.

Clients are placed in selected nodes. All loading operations can be performed by driver and takes not more than 4 to 10 minutes. This pattern is characteristic for newsstands or small FMCG shops. The calculation example considers 21 delivery points with loading operations duration from 4 to 10 minutes.

\subsection{Vehicles emission and realization time}

At this stage of research vehicle's emission is estimated for $\mathrm{CO}_{2}$. The selected vehicle was a medium 3.5 t truck with Euro 5 standard engine. Figure 3 presents convergence of genetic algorithm suiting function for example data.

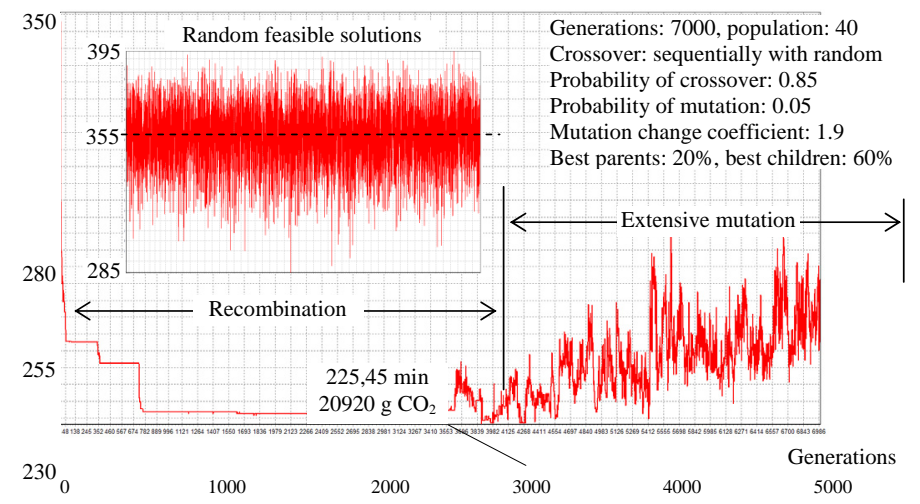

Figure 3: $\quad$ Solution found for exemplary data.

Figure 4 presents the transport network with plotted paths of rational solution found by proposed heuristics. Yellow lines mark the direction of movement determined by genetic programming, while red line is for path set by modified A-star algorithm. Numbers correspond to the following order lines. 
Visiting sequence:

$1,2,13,11,17,8$, $14,4,12,20,18$, $21,16,9,19,3,15$,

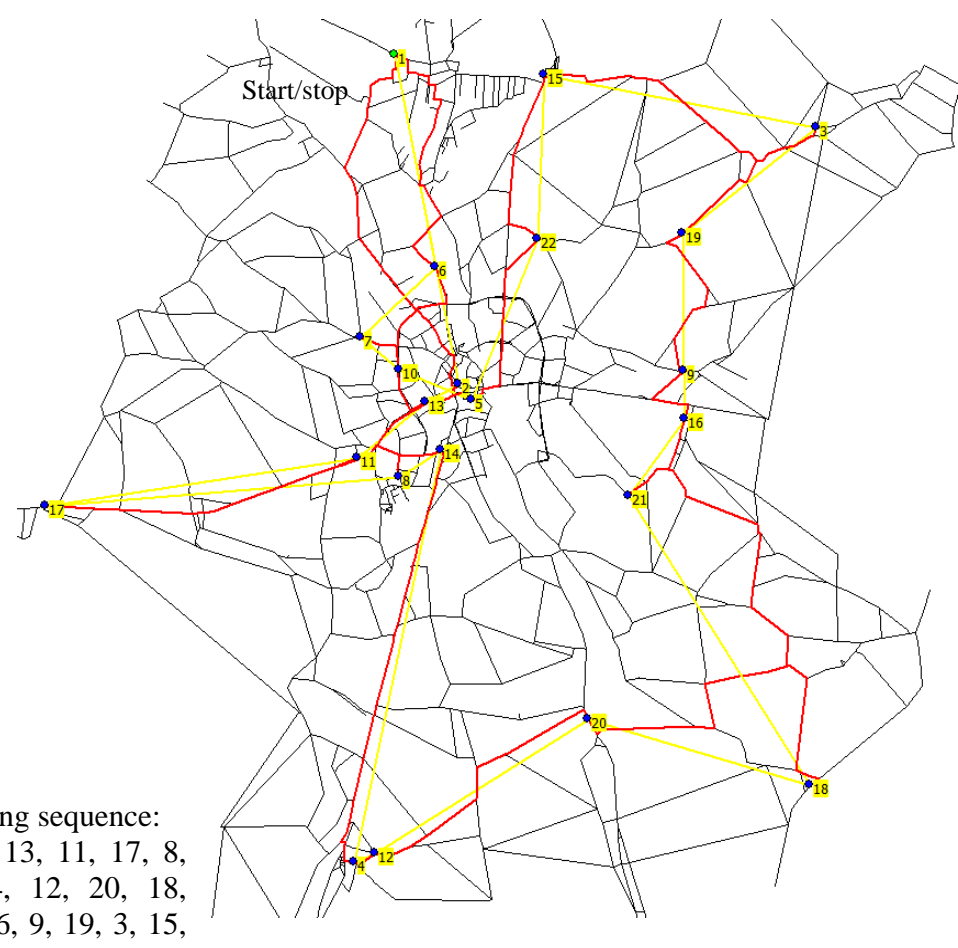

$22,5,10,7,6,1$.

Figure 4: $\quad$ Solution found for exemplary data.

\section{Conclusions and further research}

Undertaken research is the introduction to wider research program aiming in setting guidelines for designing pro-ecological transport systems. Using heuristic tools for solving class of VRP is considered effective and leads to improvement in cost efficiency or emission levels. Correlating problems of distance minimization with emission minimization allows developing wide range of tools to improve living conditions in urban areas.

Further research will embrace exact defining adjusting coefficients proposed in formal model for different types of exhaust gases components and for different types of vehicles. The emphasis should be on defining better functions describing transition costs on graph edges according to variable conditions.

\section{Acknowledgement}

The scientific research is carried out under the project "Proecological transport system designing" (EMITRANSYS) funded by the National Centre for Research and Development. 


\section{References}

[1] Malandraki, C., and M. S. Daskin. Time-Dependent Vehicle-Routing Problems: Formulations, Properties and Heuristic Algorithms. Transportation Science, Vol. 26, No. 3, pp. 185-200, 1992.

[2] Jin T., Gao J., Fu L., Ai Y., Xu X., An evaluation of improvements in the air quality of Beijing arising from the use of new vehicle emission standards. Springer Science+Business Media B. V., Environ Monit Assess, 184:2151-2159, 2012.

[3] Bektaş T., Laporte G., The Pollution-Routing Problem, Transportation Research Part B: Methodological, Vol. 45, Iss. 8, pp. 1232-1250, 2011.

[4] Figliozzi M., Vehicle Routing Problem for Emissions Minimization, Transportation Research Record: Journal of the Transportation Research Board, No. 2197, Transportation Research Board of the National Academies, Washington, D.C., pp. 1-7, 2010.

[5] Merkisz J., Bajerlein M., Kozak W., Markowski J.: The influence of CNG dissolved in the diesel fuel on the combustion process and concentration of toxic compounds in exhaust gas. 2008 SAE International Powertrains, Fuels and Lubricants Congress. 2008-01-1815, 2008.

[6] Yu L., Remote vehicle exhaust emission sensing for traffic simulation and optimization models, Transportation Research Part D: Transport and Environment, Vol. 3, Iss. 5, pp. 337-347, 1998.

[7] Kuo Y., Wang C.-C., Optimizing the VRP by minimizing fuel consumption, Management of Environmental Quality, Vol. 22, Iss. 4, pp. 440-450, 2011.

[8] Suzuki Y., A new truck-routing approach for reducing fuel consumption and pollutants emission, Transportation Research Part D: Transport and Environment, Vol. 16, Iss. 1, pp. 73-77, 2011.

[9] Kara I., Kara B. Y., Kadri Yetis M., Energy minimizing vehicle routing problem (Conference Paper), Lecture Notes in Computer Science (including subseries Lecture Notes in Artificial Intelligence and Lecture Notes in Bioinformatics), Vol. 4616 LNCS, pp. 62-71, 2007.

[10] Apaydin O., Gonullu M. T, Emission control with route optimization in solid waste collection process: A case study, Sadhana - Academy Proceedings in Engineering Sciences, Vol. 33, Iss. 2, pp. 71-82, 2008.

[11] Cordeau J.-F., Laporte G., Savelsbergh M. W. P., Vigo D., Vehicle routing Handbooks in Operations Research and Management Science, Elsevier, Amsterdam, (Chapter 6), C. Barnhart, G. Laporte (Eds.) Transp., 14, 2007.

[12] Pyza D., Transport service of a cargo consolidation centre with respect to logistics service of urban agglomerations, Urban Transport XVII: Urban Transport and the Environment in the 21st Century, Vol. 116, Wit Pr/Computational Mechanics, 2011.

[13] Jacyna M., The role of Cargo Consolidation Center in urban logistic system. Urban Transport XII, WIT Press, 2011.

[14] R. Jachimowski, J. Zak, D. Pyza, Routes planning problem with heterogeneous suppliers demand, Proceedings of 21 st International 
Conference on Systems Engineering, pp. 434-437, IEEE Computer Society, USA, 2011.

[15] Pyza D., Multicriteria Evaluation of Designing Transportation System within Distribution Sub-Systems, Logistics and Transport. Vol. 10 (1).

[16] Cornillier F., Laporte G., Boctor F. F., Renaud J., The petrol station replenishment problem with time windows, Computers and Operations Research, Vol. 36, Iss. 3, pp. 919-935, 2009.

[17] Chen H.-K., Hsueh C.-F., Chang M.-S., The real-time time-dependent vehicle routing problem, Transportation Research Part E: Logistics and Transportation Review, Vol. 42, Iss. 5, pp. 383-408, 2006.

[18] Jachimowski R., Żak J., Vehicle Routing Problem with Heterogeneous Customers Demand and External Transportation Costs, Journal of Traffic and Logistics Engineering, Vol. 1, No. 1, pp. 46-50, 2013.

[19] Jacyna M., Kłodawski M.: Model of transportation network development in aspect of transport comodality. Proceedings of 21st International Conference on Systems Engineering, pp. 341-345, IEEE Computer Society, USA, 2011.

[20] Żak J., Lewczuk K., Kłodawski M., Jachimowski R., Jacyna I., Funkcje i zadania systemu logistycznego Polski a komodalność transportu, Logistyka 4/2011, pp. 1019-1025, 2011.

[21] Lewczuk K., Jachimowski R., Jacyna I., Kłodawski M., Zadania przewozowe dla Systemu Logistycznego Polski. Prace Naukowe PW Transport z. 75 pp. 81-88, OWPW, Warsaw 2010.

[22] Lewczuk K., Wasiak M., Transportation services costs allocation for the delivery system. Proceedings of 21st International Conference on Systems Engineering, pp. 429-433. IEEE Computer Society, Las Vegas, USA, 2011.

[23] Allsop, R. E., Estimating the traffic capacity of a signalized road junction, Transportation Research /UK/, Vo. 6, Iss. 3, Pergamon Press, Inc., p. 245$55,1972$.

[24] Anthony Chen A., Yang H., Lo H. K., Tang W. H., Capacity reliability of a road network: an assessment methodology and numerical results, Transportation Research Part B: Methodological, Vol. 36, Iss. 3, pp. 225252, 2002.

[25] Lau J., Hung W. T, Cheung C. S., Observation of increases in emission from modern vehicles over time in Hong Kong using remote sensing Environmental Pollution 163, pp. 14-23, 2012.

[26] Hart, P. E.; Nilsson, N. J.; Raphael, B. A Formal Basis for the Heuristic Determination of Minimum Cost Paths. IEEE Transactions on Systems Science and Cybernetics SSC4 4 (2): 100-107, 1968. 